\title{
Radiative Heating for MSL Entry: Verification of Simulations from Ground Test to Flight Data
}

\author{
Brett A. Cruden ${ }^{1}$, Aaron M. Brandis ${ }^{2}$, Todd R. White ${ }^{3}$, Milad Mahzari ${ }^{4}$ \\ ERC Inc at NASA Ames Research Center, Moffett Field, CA, 94035 \\ and \\ Deepak Bose ${ }^{5}$ \\ NASA Ames Research Center, Moffett Field, CA, 94035
}

\begin{abstract}
The heat shield of the Mars Science Laboratory (MSL) was equipped with thermocouple stacks to measure in-depth heating of the thermal protection system during atmospheric entry. The heat load derived from the thermocouples in the stagnation region was found to be $33 \%$ lower than corresponding post-flight predictions of convective heating alone. It was hypothesized that this difference could be attributed to radiation from the shock-heated gas, a mechanism not considered in pre-flight analyses of flow fields. In order to test the hypothesis and quantify the contribution of shock-layer radiation to total surface heating, ground tests and simulations (both flow and radiation) were performed at several points along the best-estimated entry trajectory of MSL. The present paper provides an assessment of the quality of the radiation model and its impact to stagnation point heating. The impact of radiative heating is shown to account for $43 \%$ of the heat load discrepancy. Additional possible factors behind the remaining discrepancy are discussed.
\end{abstract}

\section{Nomenclature}

$\mathrm{B}_{\mathrm{i}}(\mathrm{T})=$ Planck (blackbody) function at temperature, $\mathrm{T}\left(\mathrm{W} / \mathrm{cm}^{2}-\mathrm{sr}-\mu \mathrm{m}\right)$

$\mathrm{d}=$ Shock standoff distance $(\mathrm{m})$

$\mathrm{D}=$ Shock tube diameter $(\mathrm{m})$

$\mathrm{e}_{\mathrm{i}}(\mathrm{x})=$ Emission coefficient at position $\mathrm{x}\left(\mathrm{W} / \mathrm{cm}^{3}-\mathrm{sr}-\mu \mathrm{m}\right)$

$\mathrm{L}_{\mathrm{a}}(\mathrm{x}) \quad=$ Spectral radiance at position $\mathrm{x}\left(\mathrm{W} / \mathrm{cm}^{2}-\mathrm{sr}-\mu \mathrm{m}\right)$

$\mathrm{L}_{\mathrm{i}}(\mathrm{x}, \theta)=$ Spectral radiance at position $\mathrm{x}$ at off-normal angle $\theta\left(\mathrm{W} / \mathrm{cm}^{2}-\mathrm{sr}-\mu \mathrm{m}\right)$

$n \&=$ Mass blowing rate $\left(\mathrm{kg} / \mathrm{m}^{2}-\mathrm{s}\right)$

$\mathrm{q}=$ Heat flux or irradiance

$\mathrm{T}(\mathrm{x})=$ Temperature at position $\mathrm{x}(\mathrm{K})$

$\mathrm{v} \quad=$ Velocity $(\mathrm{m} / \mathrm{s})$

$\mathrm{x}=$ Position along stagnation line or perpendicular to heat shield ( $\mathrm{cm}$ or $\mathrm{m}$ )

$\mathrm{y} \quad=$ Position along heat shield surface $(\mathrm{m})$

$\delta \quad=$ Boundary layer thickness $(\mathrm{m})$

$\lambda \quad=$ Wavelength $(\mathrm{nm})$

$\rho_{C_{x} H_{y}} \quad=$ Ablation product density $\left(\mathrm{kg} / \mathrm{m}^{3}\right)$

$\theta=$ Off-normal angle $(\mathrm{rad})$

\footnotetext{
${ }^{1}$ Research Scientist, Aerothermodynamics Branch. Associate Fellow AIAA. Contact: brett.a.cruden@nasa.gov

${ }^{2}$ Research Scientist, Aerothermodynamics Branch. Senior Member AIAA. Contact: aaron.m.brandis@nasa.gov

${ }^{3}$ Research Scientist, Aerothermodynamics Branch. Senior Member AIAA. Contact: todd.r.white@nasa.gov

${ }^{4}$ Research Scientist, Entry Systems Branch. Member AIAA. Contact: milad.mahzari@nasa.gov

${ }^{5}$ Research Scientist, Aerothermodynamics Branch. Associate Fellow AIAA. Contact: deepak.bose@nasa.gov
} 


\section{Introduction}

In 2012 NASA landed the Mars Science Laboratory (MSL), which included the Curiosity Rover, on the surface of Mars. The thermal protection system (TPS) of the entry capsule contained the MSL Entry, Descent and Landing Instrumentation (MEDLI) suite, which included the MEDLI Integrated Sensor Plugs (MISPs).[1, 2] These sensor plugs, which measured in-depth temperature profiles using thermocouples, were located at 7 positions on the forebody heat shield as shown in Fig. 1. Flying at a nominal $16^{\circ}$ angle of attack, MISP1 was located at the stagnation point, while MISP2 and MISP3 were on the leeside where peak heating was expected in the presence of the turbulent boundary layer. Flow field computations performed at several points along the best-estimated trajectory (BET) [3] yielded heating profiles that agreed well with flight measurements prior to peak heating. However, predictions at trajectory points after peak heating were found to be as much as $90^{\circ} \mathrm{C}$ lower than measurements at MISP1.[2] One hypothesis is that this difference, or discrepancy, could be attributed to shocklayer radiation.

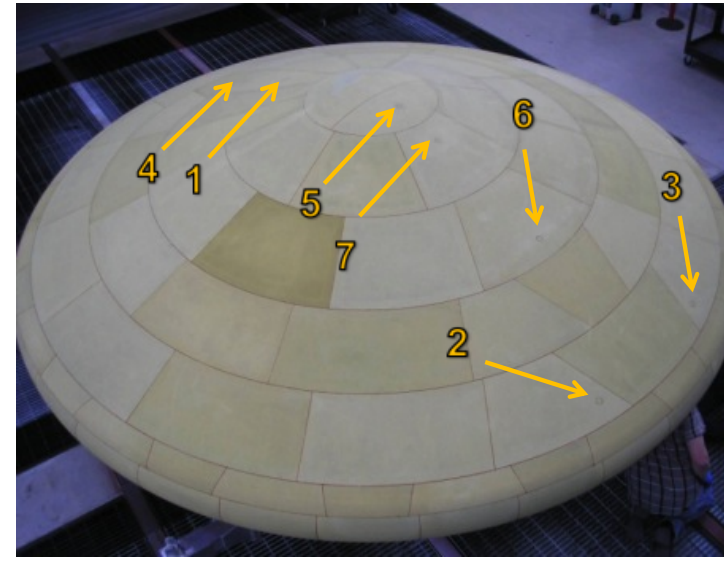

Figure 1. Image of the MSL heat shield with the location of sensor plugs labeled.

Tests conducted previously in the Electric Arc Shock Tube (EAST) facility measured mid-wave infrared (MWIR) radiation (from shock-heated $\mathrm{CO}_{2}$ ) at conditions similar to those at points along the MSL design trajectory, and the test data suggested that the magnitude of MWIR radiation was of the same order as this discrepancy.[4] The present paper provides results of new experiments conducted in the EAST facility. These new experiments replicate conditions along the MSL BET. The data obtained are compared against those from the DPLR [5] Computational Fluid Dynamics (CFD) code and NEQAIR radiation solver,[6] and an assessment is made of the accuracy of the DPLR/NEQAIR results. Estimates of radiative heating are obtained from: (a) EAST data, (b) the nominal DPLR/NEQAIR predictions, and (c) DPLR/NEQAIR models adjusted for experiment/model disagreements. These three profiles are then compared to the flight measurement and the impact assessed.

\section{Experimental Approach}

The MSL BET has been determined through analysis of the Mars Entry Atmospheric Data System (MEADS) and Inertial Measurement Unit (IMU) flight data [3] and is shown in Fig. 2 in terms of velocity and pressure/density. Five points along the MSL BET were chosen for testing in the EAST facility (described in detail in Ref. [7]), and the freestream conditions at these points are given in Table I. A gas mixture consisting of $95.8 \% \mathrm{CO}_{2}, 2.7 \% \mathrm{~N}_{2}$ and

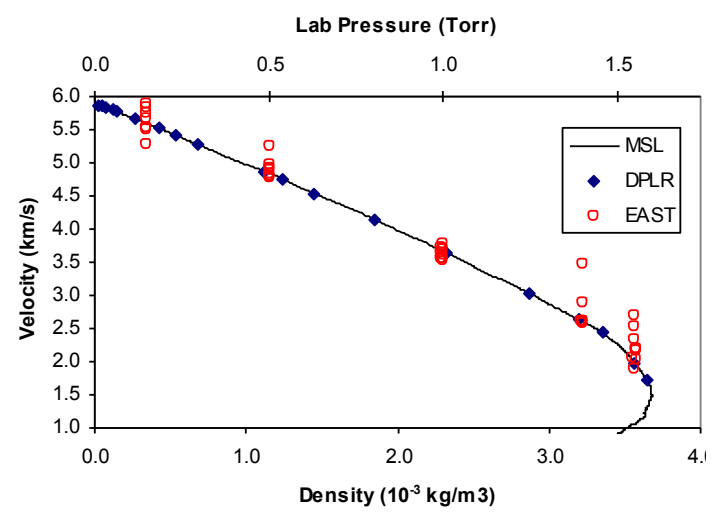

Table I. Selected entry conditions for shock tube testing

\begin{tabular}{|c|c|c|c|}
\hline $\begin{array}{c}\text { Time from } \\
\text { Entry }(\mathrm{s})\end{array}$ & $\begin{array}{c}\text { Density } \\
\left(\mathrm{g} / \mathrm{m}^{3}\right)\end{array}$ & $\begin{array}{c}\text { Velocity } \\
(\mathrm{km} / \mathrm{s})\end{array}$ & $\begin{array}{c}\text { Pressure } \\
(\text { Torr })\end{array}$ \\
\hline 63.07 & 0.34 & 5.60 & 0.15 \\
\hline 73.80 & 1.15 & 4.85 & 0.50 \\
\hline 84.90 & 2.30 & 3.61 & 1.00 \\
\hline 96.00 & 3.21 & 2.57 & 1.40 \\
\hline 104.00 & 3.55 & 2.05 & 1.55 \\
\hline
\end{tabular}

Figure 2. MSL Entry trajectory in terms of velocity and density. Test conditions in EAST and DPLR simulations are shown as points on the plot. The secondary $x$-axis indicates the pre-shock fill pressure corresponding to the required density.

Table II. Wavelength ranges of the $\mathbf{4}$ spectrometers

\begin{tabular}{|l|c|c|}
\hline Spectrometer & Range \#1 & Range \#2 \\
\hline VUV & $164-218$ & $117-173$ \\
\hline UV/Vis & $323-497$ & $190-352$ \\
\hline Vis/NIR & \multicolumn{2}{|c|}{$472-890$} \\
\hline MWIR & $3959-5403$ & $1966-3405$ \\
\hline
\end{tabular}


$1.5 \% \mathrm{Ar}$ (by volume) is used to approximate the Martian atmosphere. Since an iterative tuning procedure is required to achieve the target conditions (Table I), velocity scatter about the nominal is expected (apparent in Fig. 2). Test points with velocities outside a $\pm 0.15 \mathrm{~km} / \mathrm{s}$ band around the nominal are not included in the analysis presented in this paper.

Radiation from these shocks was measured from the Vacuum Ultraviolet (VUV) through MWIR using instrumentation described previously.[8-10] The instrumentation ranges are given in Table II. While the objective was to obtain as much spectral signature as possible, ranges were limited by the lowest resolution grating available for each spectrometer. For the MSL BET, the most important spectral ranges were identified as those corresponding to fundamental $(4.3 \mu \mathrm{m})$ and overtone $(2.7 \mu \mathrm{m})$ stretches of $\mathrm{CO}_{2}$. These data sets had to be collected in two separate shots as only one MWIR camera was used in operation. The other spectrometers are set to cover visible/near infrared (Vis/NIR: 480-900 nm), ultraviolet (UV/Vis: 200-500 nm over two shots) and VUV (120-210 nm over two shots) ranges. Below a shock speed of $4 \mathrm{~km} / \mathrm{s}$, any signal in the VUV was below the noise threshold of the camera, so the collection range was truncated at $145 \mathrm{~nm}$. For each trajectory point and wavelength range, 2-3 shots were taken within $0.15 \mathrm{~km} / \mathrm{s}$ of the target velocity. For most of the conditions here, the cameras were operated with a 2 $\mu$ s exposure time, over which the shock could move by $4-10 \mathrm{~mm}$, depending on velocity.

\section{Computational Approach}

In the analysis presented here, the flow along the stagnation streamline of a vehicle entering an atmosphere of identical composition, density and velocity as the ground-based tests is assumed to be similar to the shock tube flow. Thermochemical non-equilibrium flow computations, at several points along the BET, were performed with v4.02.2 of DPLR with the Mitcheltree-Gnoffo 8-species $\left(\mathrm{CO}_{2}, \mathrm{CO}, \mathrm{N}_{2}, \mathrm{O}_{2}, \mathrm{NO}, \mathrm{C}, \mathrm{N}\right.$, and O), 12-reaction Mars model.[11] The Martian atmosphere was modeled as $97 \% \mathrm{CO}_{2}$ and $3 \% \mathrm{~N}_{2}$ (by mass). The TPS surface was treated as a nonporous, no slip, radiative equilibrium wall with constant emissivity $(\varepsilon=0.89)$ and the Mitcheltree-Gnoffo surface catalycity model was used.[11] These assumptions are the same as those used for heating reconstruction[1, 2, 12],
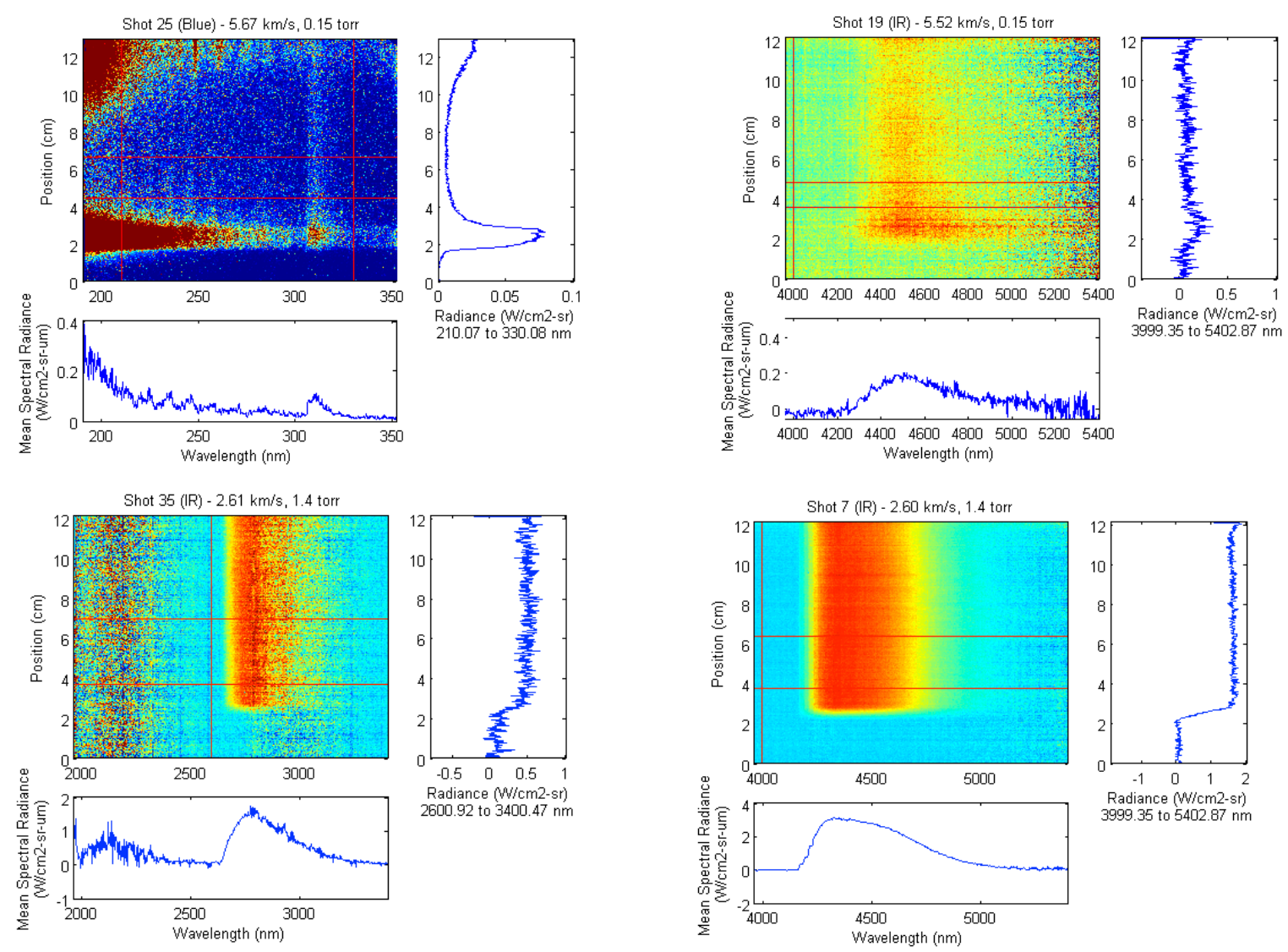

Figure 3. Measurement of radiance versus wavelength and position for representative shock conditions. (a,b) $\mathrm{t}=63 \mathrm{~s}, 5.6 \mathrm{~km} / \mathrm{s}$ (nominal), 0.15 Torr and $(\mathrm{c}, \mathrm{d}) \mathrm{t}=96 \mathrm{~s}, 2.6 \mathrm{~km} / \mathrm{s}, 1.4$ Torr. 
but differ from the design which used the conservative, but non-physical, super catalytic wall assumption.[13]

Lines of sight at locations corresponding to the stagnation point, the location of MISP1, were extracted from the DPLR solutions. NEQAIR simulations were then performed in a wavelength region from $85.5-20000 \mathrm{~nm}$ to obtain the spectral radiance along the stagnation line and the radiative heating at the stagnation point under the tangent slab approximation. NEQAIR v14.0, which contains a $\mathrm{CO}_{2}$ molecular line list based on the carbon dioxide spectral databank (CDSD-4000)[14] implemented in 2012[15], was used. This update was critical to obtaining realistic simulations of the radiative heating for the MSL entry environment.

\section{Experimental Results}

Representative results from the EAST experiments are shown in Fig. 3. Figures 3(a,b) represent the entry time of $t=63 \mathrm{~s}$, and Figs. 3(c,d) represent the entry time of $t=96 \mathrm{~s}$. The figures show radiance measurements with wavelength on the $\mathrm{x}$-axis and position on the $\mathrm{y}$-axis.

At the $\mathrm{t}=63 \mathrm{~s}$ condition, the shock is located at approximately $2 \mathrm{~cm}$ on the $\mathrm{y}$-axis. In this portion of the trajectory, measurable levels of radiation are observed in both the ultraviolet (Fig. 3a) and mid-infrared (Fig. 3b). Non-equilibrium is observed with the overshoot at the shock front, which then relaxes toward an equilibrium level of radiation behind the shock. Evidence of the end of the test is apparent in Fig. 3a where the radiance increases due to driver gas contamination at $9 \mathrm{~cm}$. The spectral signature in the ultraviolet is comprised of electronic transitions of $\mathrm{CO}$ and $\mathrm{NO}$ while the MWIR is from vibrational transitions of $\mathrm{CO}$ and $\mathrm{CO}_{2}$. While the magnitude in spectral radiance is similar for the two plots, the MWIR band spans a significantly larger wavelength range and as a consequence will contribute more significantly to the integrated spectral radiance, and hence heat flux.

At $t=96 \mathrm{~s}$ condition (Figs. 3c, and 3d), features in the ultraviolet through visible become negligible and the MWIR bands strengthen. Two bands are observed at intensities that are an order of magnitude higher than at $\mathrm{t}=63$ s. The shock shape is nearly a step function with no obvious non-equilibrium overshoot or relaxation. This is due to the higher density allowing for greater collisions and faster equilibration. For the shock tube diameter of $10 \mathrm{~cm}$, evidence of blackbody saturation is apparent for the $4.3 \mu \mathrm{m}$ band as its peak is flattened. For these two cases, no evidence of contamination or contact front arrival is observed. It should be noted that the sensitivity of the optics and spectrometer are lowered from 2-2.3 $\mu \mathrm{m}$, such that the noise in this region obscures any real signal. A reduced sensitivity is also apparent in the noise around $3 \mu \mathrm{m}$, although in Fig. $3 \mathrm{c}$ the signal is sufficiently strong to rise above this.

The summary of measurements at all five conditions is shown in Fig. 4. Here the cross-section of spectral radiance from 2-4 cm behind the shock front is averaged and presented for the UV through NIR and MWIR regions. Due to differences in noise floors between different cameras, the VUV data are not shown and the MWIR data are presented in a separate plot from the UV-NIR data. In the UV-NIR range, the radiance is seen to decrease throughout the trajectory. At the same time, the location of maximum radiance moves to higher wavelengths due to the blackbody limit changing as temperature is reduced. At $t=96 \mathrm{~s}$ and beyond, the signal is no longer distinguishable from the noise level of the camera. In the MWIR, on the other hand, radiance is increasing through the trajectory until $t=85-96 \mathrm{~s}$ where the signal reaches its maximum level before decreasing at $\mathrm{t}=104 \mathrm{~s}$. This increase is attributed to a rising $\mathrm{CO}_{2}$ density as the shock density increases and its dissociation rate decreases. Near the end of the trajectory, the lower temperature causes the radiance to begin decreasing again. The level of the MWIR radiance is orders of magnitude larger than in the visible range (note that a log scale is used in Fig. 4a). Furthermore, the features span a wavelength range that is several times larger than the visible wavelengths, so will
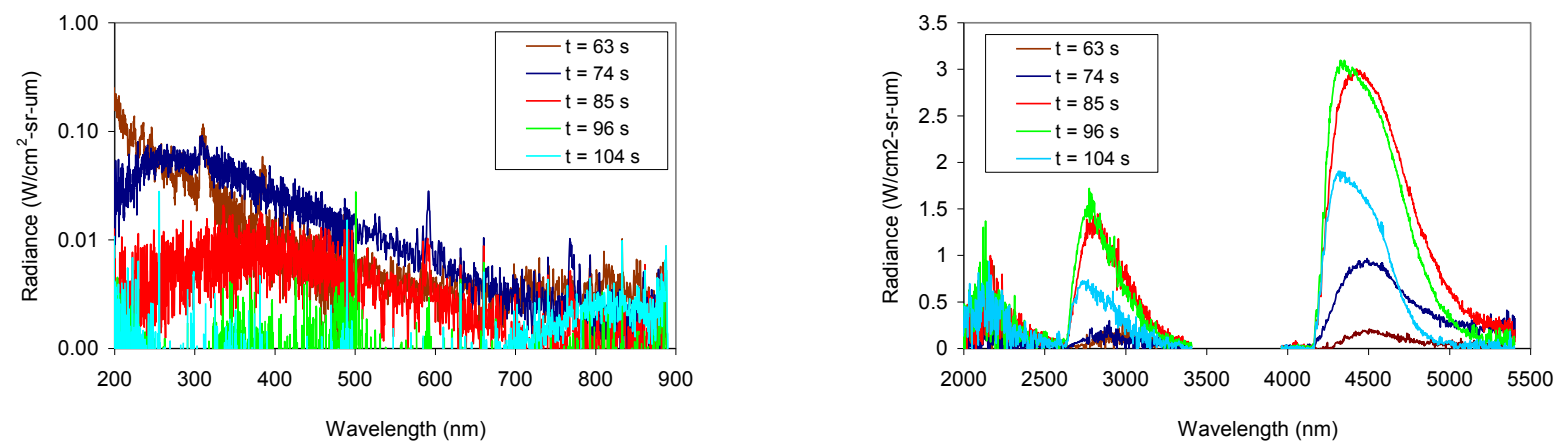

Figure 4. Radiance behind the shock at 5 trajectory points. (a) UV-NIR wavelengths, (b) MWIR wavelength. 
dominate the radiative heating.

In order to compare the shock tube measurements to the flight heating data, it is necessary to adjust for geometric factors. A procedure for doing this has been presented in [16], and is repeated here with slight modifications. On the vehicle, the radiative heating will be collected along lines of sight originating outward from the detector location on the heat shield. Under the similarity assumption, the EAST facility measurements characterize the radiance perpendicular to the vehicle's stagnation point, accumulated over a slab equal to the tube diameter. This radiance $(L)$ can be converted to a volumetric emission coefficient $(e)$ if the temperature is known:

$$
L_{\lambda}(x)=B_{\lambda}(T(x))\left(1-e^{-e_{\lambda}(x) D / B_{\lambda}(T(x))}\right)
$$

where $B_{2}(T)$ is the blackbody function at the given temperature. This emission coefficient is then used with the radiative transport equation:

$$
L_{\lambda}(d, \theta)=\int_{0}^{d} e_{\lambda}\left(\frac{x}{\cos \theta}\right)\left(1-\frac{L_{\lambda}(x, \theta)}{B_{\lambda}\left(T\left(\frac{x}{\cos \theta}\right)\right)}\right) \frac{d x}{\cos \theta}
$$

which is valid if thermal (but not necessarily chemical) equilibrium can be assumed. Here, 0 represents the shock front and $d$ is the shock stand-off distance (i.e. vehicle surface). The angular factors given follow the tangent slab assumption. Integration over angle and wavelength then yields the radiative heat flux:

$$
q=2 \pi \int_{0}^{\infty} \int_{0}^{\pi / 2} \sin \theta \cos \theta L_{\lambda}(d, \theta) d \theta d \lambda
$$

To perform this computation, two different temperature profiles have been assumed. The first uses the profile of DPLR flow field computations using the Mitcheltree-Gnoffo chemistry.[11] For the integration in equation (2), $d$ is assumed to end at the boundary layer edge; the absorption predicted in the boundary layer is $<3 \%$ and is therefore neglected. The second approach uses the EAST data itself by calculating the blackbody temperature that bounds the data. In this case the integration is carried through the value of $d$ extracted from the DPLR flow field solution. The temperature profile obtained this way is extrapolated using a best-fit exponential decay toward equilibrium. The integration is carried through the shock stand-off distance derived by CFD. The intent of exploring this method of temperature determination is to separate model dependencies from a purely experimental determination of heat flux, although the result is still dependent upon the shock standoff distance derived by CFD.

The evaluation of equation (2) for $\theta=0$ at different values of $d$, using DPLR profile is shown in Fig. 5 for two conditions at different wavelength ranges. At the low pressure condition (Fig. 5(a)), the non-equilibrium contribution from the VUV is initially apparent but then decreases as it is reabsorbed in the shock layer. Radiation from the 2.7 and $4.3 \mu \mathrm{m}$ bands increases linearly with position, as would be expected for an optically thin radiator. Later in the trajectory (Fig. 5(b)), the $4.3 \mu \mathrm{m}$ band is seen to quickly rise and then slowly increase beyond the shock
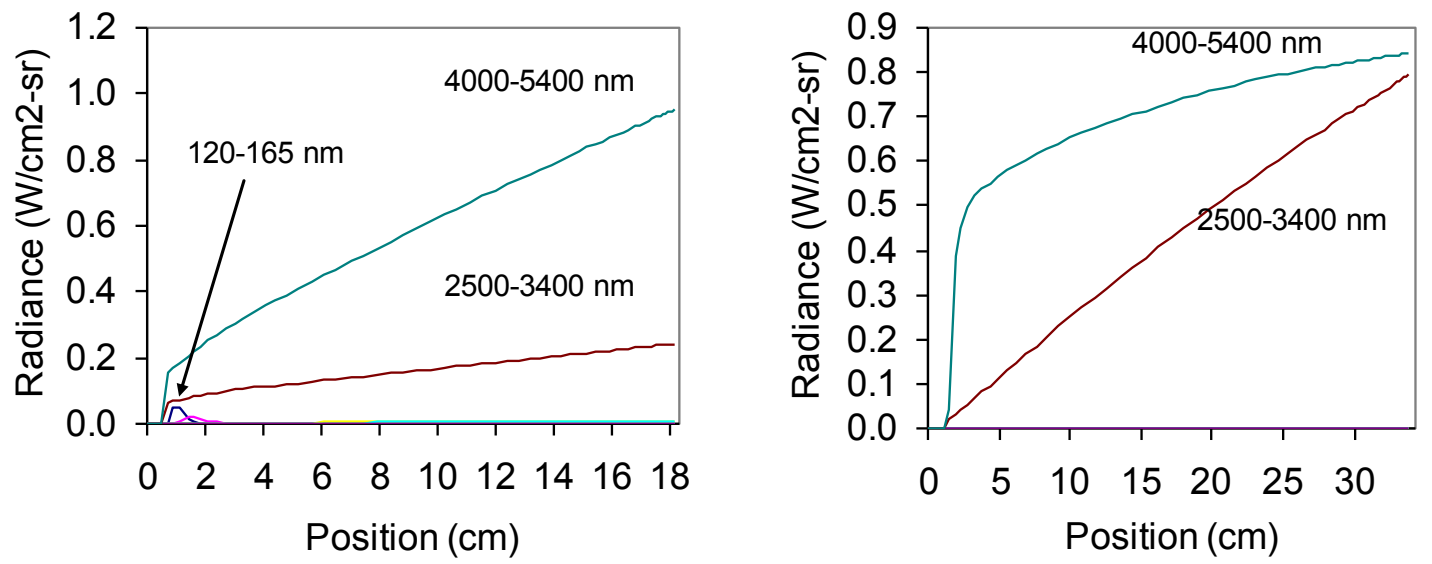

Figure 5. Radiance accumulated over position from EAST data analysis. (a) $t=74 \mathrm{~s}$, (b) $t=104 \mathrm{~s}$. 


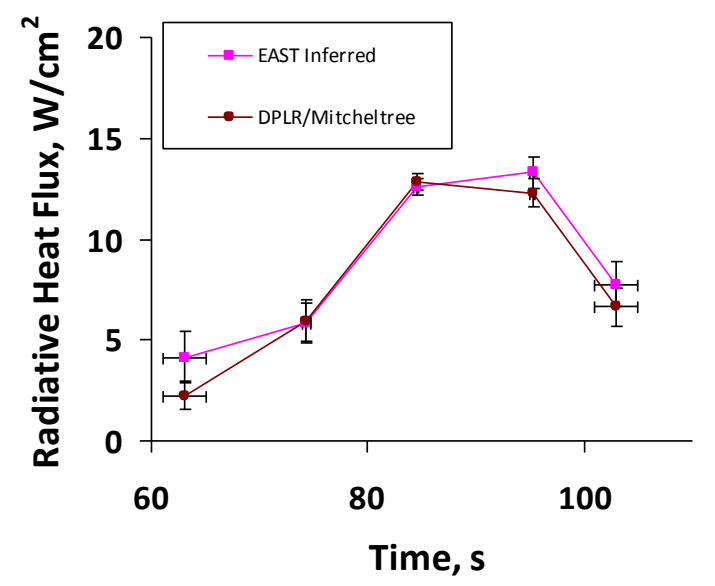

Figure 6. Radiative heat flux inferred from EAST experiments. The different curves represent the heat fluxes obtained assuming temperature profiles obtained through different methods. front. The plateau is attributed to the optical thickness of the band. The radiance increases slowly at longer distance as radiance from the outer edges of the band accumulates to their blackbody limits. The $2.7 \mu \mathrm{m}$ band, on the other hand, increases linearly, indicating it remains optically thin over $35 \mathrm{~cm}$.

The heat flux obtained from these approaches is shown in Fig. 6. The error bars represent the variation (standard deviation) between tests at similar conditions, weighted by the relative contribution of the different wavelength ranges. The $\mathrm{x}$-error bars indicate the range of velocities analyzed and where they correspond to in MSL entry time. The DPLR solutions are obtained at slightly different conditions than those in Table I but are always contained within the $\mathrm{x}$-error bar range (see Fig. 2 ). Given the relative similarity of the two curves, it appears the experimentally derived heat flux is reasonably determined based on the DPLR-obtained temperature profiles. However, this may be re-examined in the future with alternative kinetic models.

\section{Radiative Heating Prediction}

To assess the radiative heating profile, predictive tools are required. The results of this section are discussed first in terms of the comparison of predictive simulations to the EAST data and then in terms of the radiative heating profile for MSL entry. Figure 7 shows a comparison of the radiance versus position from the $4.3 \mu \mathrm{m}$ band in the EAST data and for the DPLR/NEQAIR stagnation line simulation corresponding to the MSL entry time of $84 \mathrm{~s}$ (velocity of approximately $3.7 \mathrm{~km} / \mathrm{s}$ ). Excellent agreement is obtained, and it is clear from this plot that neither CFD nor experiment has sufficient time to relax to equilibrium. Therefore, an assessment of the equilibrium radiation prediction is not generally possible for these tests.

Due to limitations in test time and the physical window length, the EAST experiment cannot replicate the radiance over the entire shock stand-off distance. Therefore, in order to assess the level of agreement between EAST and the simulation results, the temporal traces are integrated $\pm 2 \mathrm{~cm}$ from the shock front location. This integrated value is here referred to as the "Absolute Nonequilibrium Radiance," as discussed in our previous publications.[16, 17] This comparison is used to provide a bounding factor for the accuracy of the simulation, which is otherwise completely independent of the EAST data. This comparison is shown for the two dominant spectral regions in Fig. 8 (a). Near peak radiative heating, these two spectral regions comprise approximately $90 \%$ of the total radiative heat flux. Though the data is displayed as a function of velocity, the pressure is also changing throughout the test points as represented by Fig 2 . As suggested by Fig. 7, good agreement is obtained over the 3959 to $5000 \mathrm{~nm}$ range. A slight shift in velocity space is apparent - increasing the experimentally measured velocity by $0.2 \mathrm{~km} / \mathrm{s}$ would result in near perfect agreement. The accuracy of velocity measurements, however, is estimated at better than $0.05 \mathrm{~km} / \mathrm{s}$. While in previous publications, we have postulated that the affect of shock deceleration may appear similar to an increased velocity[18, 19], this effect is not expected to be noticeable within 2 $\mathrm{cm}$ of the shock front. The alternative would be to attribute the difference to the kinetic model, as we have explored in previous publications[10]. As shown in Ref. [10],

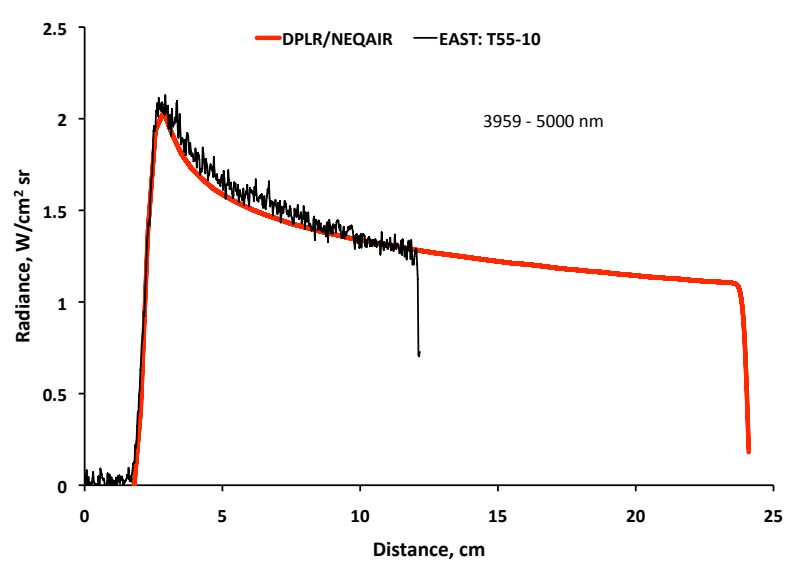

Figure 7. Spatial comparison of DPLR/NEQAIR with EAST. 
different models match the data at different velocities, the result of which would be a shift when the data is compared to only one model as a function of velocity. Other works have explored adjustments to the chemical kinetics at higher velocity, and shown significant impact on radiative heating in non-equilibrium. $[17,19,20]$
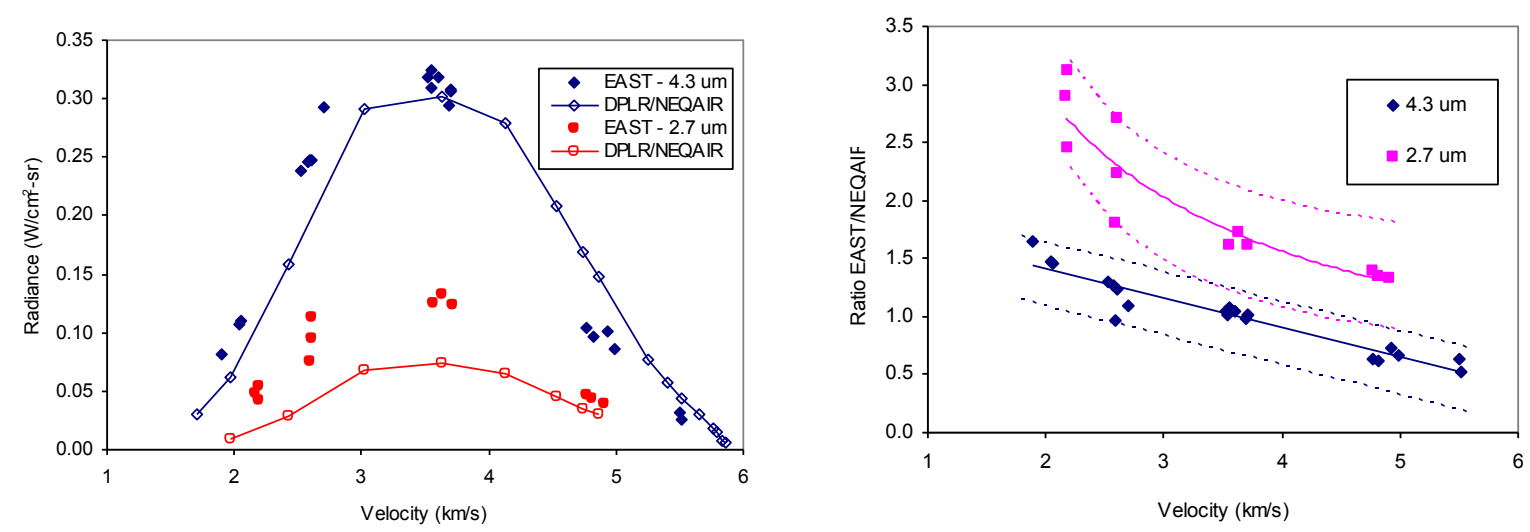

Figure 8. (a) Comparison of DPLR/NEQAIR simulations with EAST for dominant spectral ranges of 2600$3404 \mathrm{~nm}$ and 3959-5000 $\mathrm{nm}$. (b) Margin factors for scaling radiative data to experiment. The solid line displays the nominal scaling factor while the dotted lines give the band of scaling factors to encompass all data points.

The simulations under-predict the 2600 to $3404 \mathrm{~nm}$ wavelength range by as much as a factor of three, as was shown in our previous publication for similar conditions.[10] The underprediction may indicate that the $\mathrm{CO}_{2}$ dissociation rate at the shock front is too fast with the Mitcheltree kinetics. The $4.3 \mu \mathrm{m}$ band would be less sensitive to such an overprediction due to its optical thickness. However, it was also noted that this band could exceed even the frozen limit in some cases, suggesting possible issues with the spectroscopic database at high temperature. It should be noted that this band is well predicted in microwave plasmas in the temperature range $2000-3000 \mathrm{~K},[21,22]$ so that database related issues should only apply when the frozen shock temperatures are larger than this.

Regardless of the reason for the mismatch to experiment, the radiative heating prediction can be assigned an uncertainty that is carried through to the margin determination for heat shield sizing purposes. Here, we quantify the uncertainty by the level of disagreement of the prediction to shock tube data. This is expressed as a ratio in Fig. 8(b) for both the 2.7 and $4.3 \mu \mathrm{m}$ band, which displays the disagreement for discrete points and best fit lines through the ratio. A further band can be placed upon the ratio to encompass all data points, as shown by the dotted lines. Though the $2.7 \mu \mathrm{m}$ band is significantly underpredicted, it is generally less important in terms of the overall radiative heating, being between $15-25 \%$ as strong as the $4.3 \mu \mathrm{m}$ band. The overall uncertainty factor is then weighted by the relative contribution of each band to the total heat flux. The NEQAIR/DPLR prediction can then be scaled by this factor to obtain a "corrected" NEQAIR heat flux.

Figure 9 shows the radiative heating as predicted by DPLR/NEQAIR for MISP1. The radiative heating peaks at approximately $12 \mathrm{~W} / \mathrm{cm}^{2}$, and later in the trajectory when compared to peak convective heating. Also shown is the corrected NEQAIR heat flux and experimentally derived heat flux discussed in Section IV. The experimentally derived heat flux is greater than the NEQAIR prediction at all times but displays the same peak location at approximately $14 \mathrm{~W} / \mathrm{cm}^{2}$. The corrected NEQAIR data are lower than the experimentally derived flux during the rising pulse and is higher at the peak and later times. The difference in the rising pulse is attributed mostly to noise in the measurement of the $2.7 \mu \mathrm{m}$ band, leading to an overestimate of radiative heat flux in the EAST

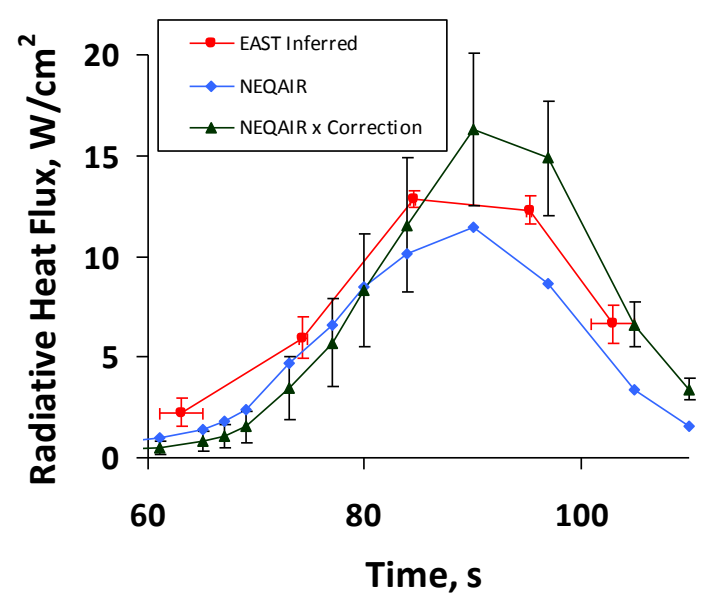

Figure 9. DPLR/NEQAIR estimated radiative heating for MISP1.

7

American Institute of Aeronautics and Astronautics 
(a)

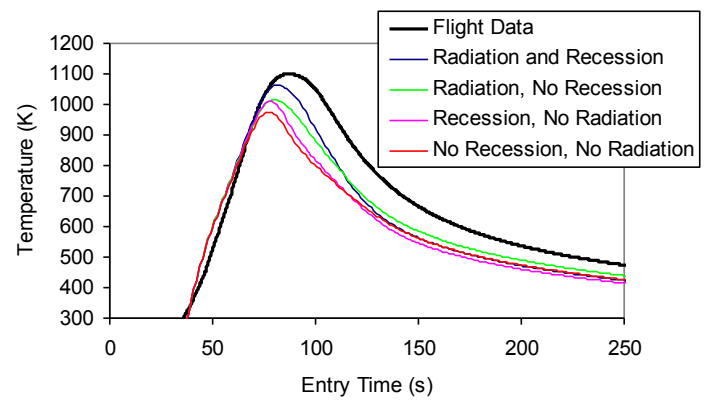

(b)

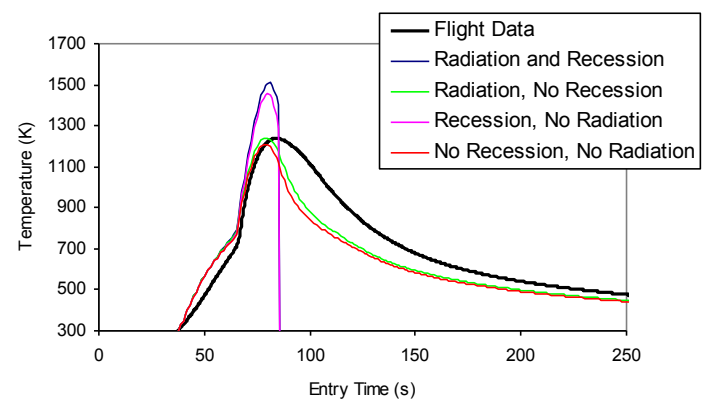

Figure 10. Combined effect of recession and radiation on surface thermocouple readings at (a) Stagnation Point (MISP1) and (b) leeside (MISP2).

inferred heat flux. The difference at peak and cool down comes from using the correction factor derived within 2 $\mathrm{cm}$ of the shock front across the entire shock thickness, which may make the corrected heat flux overly conservative. The error bars on the corrected heat flux originate from the bounding bands given in Fig. 8(b) and are as large as 4 $\mathrm{W} / \mathrm{cm}^{2}$ at peak heating. This error is conservative, however, as it is driven more by the disagreements at lower velocity than at the peak condition.

\section{Comparison to Flight Data}

The heating profiles using only convection and convection with the nominal NEQAIR predictions are processed through the FIAT[26] code both with and without recession enabled for locations corresponding to MISP1 (stagnation line) and MISP2 (far lee-side) in order to compare to the flight data. Figure 10 shows the resulting temperature profiles at the top thermocouple location, which is nominally $2.54 \mathrm{~mm}(0.10 ")$ below the TPS surface. Including recession has substantial impact on the surface thermocouple reading as the surface is brought closer to the thermocouple via recession. At MISP1, the prediction excluding radiation and recession is underpredicted by about $123 \mathrm{~K}$. Including recession increases the top thermocouple temperature by about $33 \mathrm{~K}$, but does not significantly alter the heating profile. Including radiation both increases the peak temperature and broadens the overall shape of the curve. When radiation is combined with recession, the effect is more than simply additive as the radiative heating induces additional recession. Here the peak temperature is $35 \mathrm{~K}$ less than the flight data and the temperature peak is shifted later in time. In the case of MISP2, the flight reading is $139 \mathrm{~K}$ larger and the convective heating is several times greater.[2] Because the heating is larger overall, the inclusion of radiation, which has similar magnitude at MISP2 in comparison to MISP1, has smaller relative impact. The peak temperature is closely matched even with no recession, though the temperature profile is underpredicted in the cool-down phase. Including nominal recession overpredicts the peak temperature and leads to thermocouple burnout. Therefore it is presumed that the equilibrium recession model is inaccurate. Such interpretation is supported through previous arc jet tests performed in air.[23]

A more in depth interpretation of the flight data requires some understanding of the material response mechanisms. A simplified heat balance diagram is shown in Fig. 11. The incoming convective and radiative heating are balanced by thermal conduction into the material, ablative removal and thermal surface re-radiation. The ablative component includes both chemical reactions which, being exothermic, add heat to the material, and the transport of ablation products out of the material, which removes heat. In the general formulation of the heat balance equations, it is not possible to separate convective heating from the environment from convective removal of ablation products. Only the in-depth conduction is measured in flight, and other terms must be inferred through inverse analysis.[24, 25]

The inverse analysis essentially iterates backward through the material response code to obtain a surface heating profile that matches in-depth thermocouple measurements. The heating profile obtained is unable to separate heating terms attributable to environmental factors (i.e. convective and radiative heating) from heating due to ablation and surface chemistry. The inverse analysis therefore obtains a heating that is inclusive of both environmental heating and ablative removal. The remaining terms in the heat balance, in-depth conduction and reradiation, are determined by the temperature profile in the material. Therefore, the following equalities hold:

$$
\mathrm{q}_{\text {inverse }}=\mathrm{q}_{\text {env }}-\mathrm{q}_{\mathrm{abl}}=\mathrm{q}_{\mathrm{re}-\mathrm{rad}}+\mathrm{q}_{\mathrm{cond}}
$$




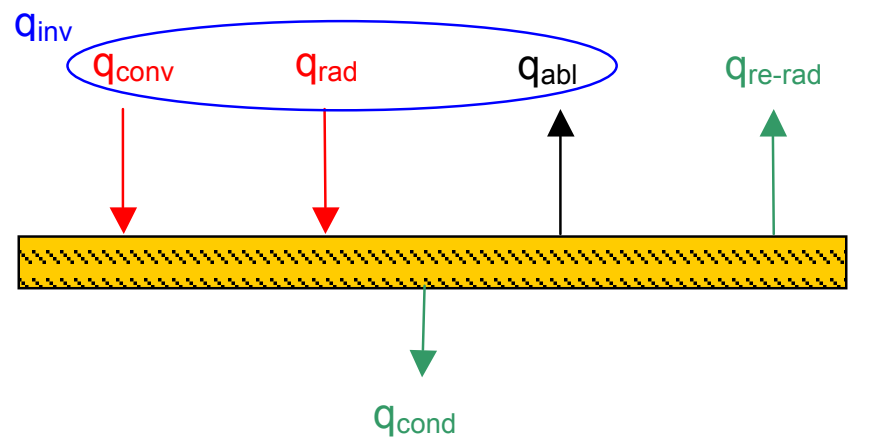

Figure 11. A simplified diagram of heat balance terms for material response. Terms in red originate from the environment. Terms in green are determined by material temperature profile. The circled terms comprise the flight (inverse) derived heat flux.

In theory, the inverse analysis should also predict a recession profile, but in practice this would often fail. The recession profile was therefore used as input to the inverse process to solve for the derived heating. This recession profile is obtained by running the nominal environmental heating through FIAT as a direct (forward) simulation. As a result, any changes to the nominal environment require re-evaluation of the inverse analysis with the new recession profile. The data extracted from inverse analysis are assigned a confidence interval via Monte Carlo analysis[24] of the various input parameters. For MISP1, a recession profile uncertainty is also incorporated into the confidence intervals by allowing the recession rate to be scaled by a constant, which is given a flat distribution between 0.66-1.28 (these being the range of errors in Ref [23]) in the Monte Carlo analysis. This was not possible on MISP2 as thermocouple burnout precludes a multiplier larger than 0.67 . The confidence intervals cannot account for inaccuracies in the way the model is constructed, such as the assumption of equilibrium surface composition. Other epistemic uncertainties may also impact this assessment.

As an initial comparison of the inferred and predicted heat flux, we estimate the environmental heat flux as the sum of cold wall convective heat flux with the radiative heat flux (weighted by material absorptivity). The difference between the heat flux from inverse analysis and this environmental flux will then represent the combination of ablative heat flux and model error. Modeling errors can originate in either the environment prediction or material response models (predictive or inverse). A negative value in this context is physically inconsistent with ablation so must be assigned to modeling error. Since the material response code cannot predict negative ablation, the error is assigned to the environment prediction. More specifically, it indicates that a heating mechanism is absent in the environment prediction. Figure 12 shows the difference between the environment and inverse heat flux, both with and without radiation included. If the model predictions were correct, the quantity shown would represent the heat lost to ablation. When radiation is excluded, the difference of these two quantities becomes negative near $\mathrm{t}=87 \mathrm{~s}$. Including the nominal radiation model eliminates most of the negative region until $t=106 \mathrm{~s}$, indicating that this analysis is physically more consistent by including radiation. As the difference still goes negative when radiation is included, a combination of more heating and/or model error would be required to make the models completely consistent. It should be noted that the radiative heating had to be extrapolated beyond $\mathrm{t}=$ $106 \mathrm{~s}$, which suggests that this extra heating could be due to radiation and thus continue to be relevant much further

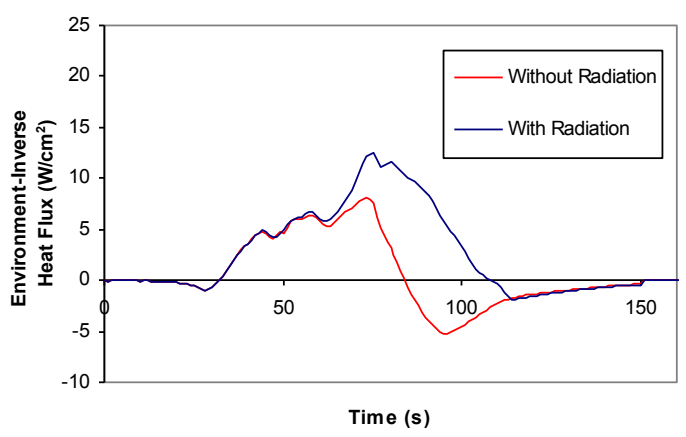

Figure 12. The difference between inverse derived heat flux and cold wall heat flux as a function of entry time, with and without the inclusion of radiation. For a perfect model, this difference will be the energy removed by ablation. into the trajectory.

Finally, the three profiles from Fig. 9 are processed through the FIAT material response code, along with convective heating terms (film coefficient, recovery enthalpy) to obtain a heating profile which can be compared to the inverse analyzed flight data. The profiles of Fig. 9 cannot simply be added to the convective heating as the heat 
(a)

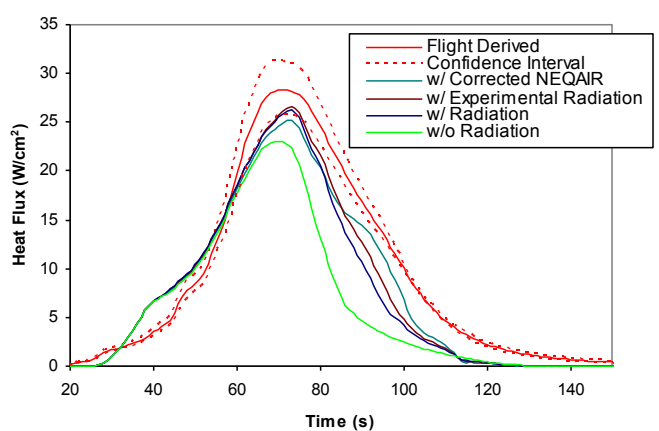

(b)

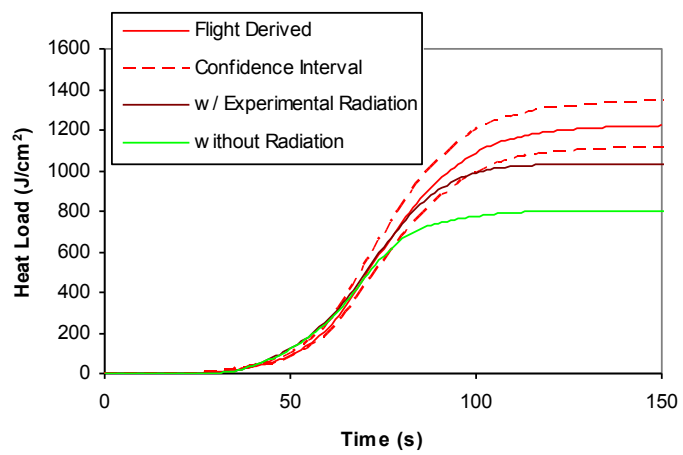

Figure 13. (a) Comparison of heat flux profiles obtained with the three radiation analyses and with convection only. Flight data from inverse analysis is also shown with its confidence interval. (b) Heat Load determined from flight data and predicted with and without radiation terms.

shield responds by rejecting between $30-50 \%$ of the additional heat flux by thermal radiation, reflection/scattering, and ablation/blowing. The curves shown in Fig. 13(a) represent the heat flux corrected by FIAT's prediction of the latter two effects, in order to be compared to the inverse analysis. The surface thermal radiation is calculated and explicitly accounted for in both the forward and inverse FIAT heat balances, so is not included in Fig. 13. The inclusion of radiation is seen to increase the peak heat flux to nearly coincide with the lower limit of the confidence interval. Additionally, the peak location has shifted in time to agree with the flight data. At later times in flight, however, the heat flux remains underpredicted by more than the bounds of the confidence interval. The amount of underprediction is approximately halved versus convection alone. The three different radiation curves are qualitatively similar, though the corrected NEQAIR profile shows an inflection, which is not apparent in the other data sets. This is attributed to an overly conservative correction factor at late times rather than indicating an improved prediction of heat flux.

The impact in terms of heat load is shown in Fig. 13(b). Heat load, as the integral of heat flux, is a more relevant quantity in terms of heat shield sizing. The heat load shown here includes both ablative and environment terms. The nominal heat load estimated from the flight sensors is $1200 \mathrm{~J} / \mathrm{cm}^{2}$ with a $95 \%$ confidence interval of \pm 100 $\mathrm{J} / \mathrm{cm}^{2}$. The heat load found from convection alone underpredicted this heat load by $400 \mathrm{~J} / \mathrm{cm}^{2}$, or $33 \%$. Including radiation reduces the error to $230 \mathrm{~J} / \mathrm{cm}^{2}$, or $19 \%$. Also shown are the heat loads obtained using the experimentally derived and corrected NEQAIR heating. These display errors of $16 \%$ and $14 \%$, respectively. This error is still greater than the confidence interval, so would need to be carried as a margin factor in future sizing exercises.

\section{Discussion}

While inclusion of radiative heating is shown to improve the agreement to flight data and is based upon physically verified mechanisms, an additional discrepancy remains. Suggested areas for future work will be discussed here. The remaining discrepancy may arise from errors in either the environment or material response models. One possible source of error is in the use of an equilibrium, rather than finite rate, ablation model in the material response computations.[23] The inclusion of finite-rate ablation should provide a more accurate calculation of wall enthalpy. If the wall enthalpy is increased, the corresponding energy removal via ablation will be reduced in the forward analysis. This would in turn increase the predicted heat flux and bring the prediction into better agreement with the data. The finite rate model will also alter the recession calculation.

The recession calculation appears to be favorable in terms of predicting the temperature profiles at MISP1, however is too large at MISP2. It is therefore of interest to determine whether the recession model may have performed better on the stagnation point than on the leeside of the vehicle. During the arc-jet testing for MSL qualification, an under-prediction of recession was realized in shear, which is the opposite of the current observation.[27] This mechanism is currently understood to be specific to the test configuration and not expected to be observed in flight.[28] The tendency of equilibrium recession models to overpredict recession has been previously examined.[23] Close examination of the data in Ref. [23] shows the recession underprediction to decrease monotonically with enthalpy but is not conclusively correlated with either pressure or heat flux. The enthalpy for MSL is comparable to the range where the equilibrium model is expected to be adequate. However, due to the difference in the test gas used for the arc-jet experiments (Air) and the atmosphere experienced during the MSL entry (primarily $\mathrm{CO}_{2}$ ), this value may not be significant. The finite-rate recession model necessary to confirm 
this is still under development for Martian atmospheres. Another possibility is that an overprediction of turbulent heating at MISP2 could result in an excessive recession prediction. The fact that the heating model reaches the peak temperature in the absence of any recession suggests this may be occurring.

It is known that recession rate is correlated to atomic oxygen concentration in the boundary layer. Examination of the CFD profiles show the atomic oxygen concentration at the MISP2 boundary layer edge to be lower than equilibrium by about $15 \%$, while it is within $5 \%$ of equilibrium at MISP 1 . This could cause greater overprediction at the lee-side, but is unlikely to fully explain the recession discrepancy. One possibility not yet examined is that ablation products produced upstream may be entrained in the boundary layer, thereby displacing atomic oxygen. To check the feasibility of this, we estimate the average ablation product density in the boundary layer as:

$$
\left\langle\rho_{C_{x} H_{y}}\right\rangle=\frac{\int_{0}^{\delta} \dot{m} d y}{\int_{0}^{\delta} v d x}
$$

The numerator is estimated by taking the blowing rates from FIAT at MISP1 and MISP2 and multiplying the average by their separation, equivalent to having a linearly varying ablation rate over the heat shield. The denominator is found from integrating the DPLR solution over the boundary. In doing so, the mean ablation product density in the boundary layer is estimated at $0.015 \mathrm{~kg} / \mathrm{m}^{3}$, which is more than half of the mean density through the boundary layer. While this should be taken as no more than an order of magnitude analysis, it shows that it is plausible for the boundary layer composition to be sufficiently altered so as to impact recession rate. Coupled fluid dynamic/material response codes (currently under development [29]) would be required to correctly account for this phenomena.

\section{Conclusions}

The underprediction of the flight heating profile in the recent MSL entry has been investigated in terms of the contribution from radiative heating, which was not included in the original analysis. The present work considers the possibility that vibrationally hot $\mathrm{CO}_{2}$ may contribute sufficient emission to impact the heat flux and heat load during entry. To determine this contribution, the radiative heating is both simulated using the NEQAIR code with DPLR CFD flow field and is measured in the Electric Arc Shock Tube facility. Radiation in the VUV through Near Infrared is shown to be negligible in comparison to the mid-infrared radiation. The majority of the radiative heat flux is due to the two major bands of $\mathrm{CO}_{2}(4.3 \mu \mathrm{m}$ and $2.7 \mu \mathrm{m})$, both of which have been characterized in this study. Comparing the NEQAIR simulations to the EAST data indicate the $4.3 \mu \mathrm{m}$ band to be matched to within a velocity shift of $0.2 \mathrm{~km} / \mathrm{s}$. This comparison suggests that the disagreement is most likely related to the chemical kinetics model used in the CFD solver. The $2.7 \mu \mathrm{m}$ band is underpredicted by as much as $3 \times$, with the error decreasing at higher velocity. The reason for this underprediction is presently unclear, and might be related to a combination of inadequacy of the spectral database and difficulties in experimentally measuring this region of the spectrum.

The radiative heat flux expected based on the MSL trajectory is then generated in three different ways. First, NEQAIR is used to directly simulate the radiative heating magnitude. Second, geometric arguments are used to infer a radiative heating directly from the EAST data, although this magnitude is still dependent upon the DPLR temperature profile. Finally, a correction factor for NEQAIR is determined based on EAST simulations and applied across the flight profile. These three methods yield peak radiative heat fluxes of 12,14 and $16 \mathrm{~W} / \mathrm{cm}^{2}$, respectively, and peaked later in time than the convective pulse. These heat flux profiles have been used with the material response code to determine a heating that may be compared to that obtained via inverse analysis of the flight data, and is shown to improve the agreement to the flight profile. The error in heat load is reduced from $33 \%$ to $19 \%$ by inclusion of radiation. This error is still outside of the confidence intervals of the flight data suggesting that there are other model deficiencies. It is speculated that using a finite-rate ablation chemistry model, rather than an equilibrium one, will improve the agreement.

It is shown that neglecting recession at the stagnation point exacerbates the disagreement between flight and predictive models at MISP1. However, at MISP2, the nominal recession profile overpredicts the temperature profile and leads to TC burnout. Including radiation at the leeside MISP increases the temperature but not sufficiently to impact the disagreement. Using a finite rate ablation model is expected to improve the recession prediction and reduce the disagreement. This may also change the energy lost to ablation products by altering the wall enthalpy, thus increasing the predicted heating. It is further proposed that ablation products produced along the heat shield will displace atomic oxygen in the boundary layer, which can also reduce recession and alter the heat transfer coefficient. 
On the basis of this work, several areas of continued research are recommended for resolving the discrepancy in heating measurements. First, alternate kinetic models may improve the radiative predictive capability, and possibly alter the convective heating terms. Alternate catalycity models may also have impact on the convective prediction. Furthermore, improvement to the material response models to include finite-rate chemistry is required. Equilibrium ablation/recession models are known to be inaccurate in certain regimes and may explain at least part of the remaining discrepancy. Further investigation on the role of ablation products in determining the boundary layer composition is needed, as this will impact the recession rate differently across the heat shield. A coupled material response/fluid dynamics model should have significant impact in this direction.

\section{Acknowledgments}

The authors thank NASA's Entry Systems Modeling (ESM) project and MEDLI for their support of this work. Drs. Cruden, Brandis, Mahzari and White are supported through the NNA10DE12C contract between NASA Ames Research Center and ERC, Inc.

\section{References}

1. White, T. R., Mahzari, M., Bose, D., and Santos, J. A., "Post-flight Analysis of Mars Science Laboratory's Entry Aerothermal Environment and Thermal Protection System Response," AIAA Paper 2013-2779.

2. Bose, D., White, T., Mahzari, M., and Edquist, K., "Reconstruction of Aerothermal Environment and Heat Shield Response of Mars Science Laboratory," Journal of Spacecraft and Rockets, Vol. 51, No. 4, 2014, pp. 1174-1184.

3. Karlgaard, C., Kutty, P., Shidner, J., Schoenenberger, M., and Munk, M., "Mars Entry Atmospheric Data System Trajectory Reconstruction Algorithms and Flight Results," AIAA 2013-0028.

4. $\quad$ Cruden, B. A., Prabhu, D., and Martinez, R., "Absolute Radiation Measurement in Venus and Mars Entry Conditions," Journal of Spacecraft and Rockets, Vol. 49, No. 6, 2012, pp. 1069-1079.

5. Wright, M. J., Candler, G., and Bose, D., "Data-Parallel Line Relaxation method for the Navier-Stokes Equations," AIAA Journal, Vol. 36, No. 9, 1998, pp. 1603-1609.

6. Whiting, E., Park, C., Liu, Y., Arnold, J., and Paterson, J., "NEQAIR96, Nonequilibrium and Equilibrium Radiative Transport and Spectra Program: User's Manual," NASA RP-1389, 1996.

7. Cruden , B. A., "Absolute Radiation Measurements in Earth and Mars Entry Conditions ", Technical Report RTO-ENAVT-218, NATO.

8. Greenberg, R. B., Cruden, B. A., Grinstead, J. H., and Yeung, D., "Collection optics for imaging spectroscopy of an electric arc shock tube," Proccedings of the SPIE, Novel Optical Systems Design and Optimization XII. Vol. 7429, $2009,74290 \mathrm{H}$.

9. Cruden, B. A., Martinez, R., Grinstead, J. H., and Olejniczak, J., "Simultaneous Vacuum Ultraviolet through Near IR Absolute Radiation Measurement with Spatiotemporal Resolution in an Electric Arc Shock Tube," 41st AIAA Thermophysics Conference. AIAA, San Antonio, TX, 2009, AIAA 2009-4240.

10. Cruden, B. A., Prabhu, D. K., and Brandis, A. M., "Measurement and Characterization of Mid-wave Infrared Radiation in CO2 Shocks," AIAA Paper 2014-2962.

11. Mitcheltree, R. A., and Gnoffo, P. A., "Wake Flow About a MESUR Mars Entry Vehicle," AIAA-94-1958.

12. Edquist, K. T., and Hollis, B. R., "Mars Science Laboratory Heatshield Aerothermodynamics: Design and Reconstruction," AIAA Paper 2013-2781.

13. Edquist, K., Dyakonov, A., Wright, M., and Tang, C., "Aerothermodynamic Design of the Mars Science Laboratory Heatshield," AIAA 2009-4075.

14. Tashkun, S. A., and Perevalov, V. I., "CDSD-4000: High-resolution, high-temperature carbon dioxide spectroscopic databank," Journal of Quantitative Spectroscopy and Radiative Transfer, Vol. 112, 2011, pp. 1403-1410.

15. Palmer, G., and Cruden, B., "Experimental Validation of $\mathrm{CO}_{2}$ Radiation Simulations," AIAA Paper 2012-3188.

16. Cruden, B. A., "Radiance Measurement for Low Density Mars Entry," AIAA Paper 2012-2742.

17. Brandis, A. M., Johnston, C. O., Cruden, B. A., and Prabhu, D. K., "Investigation of Nonequilibrium Radiation for Mars Entry," AIAA Paper 2013-1055.

18. Brandis, A. M., Cruden, B. A., Prabhu, D., Bose, D., McGilvray, M., and Morgan, R. G., "Analysis of Air Radiation Measurements Obtained in the EAST and X2 Shocktube Facilities," 10th AIAA/ASME Joint Thermophysics and Heat Transfer Conference. Chicago, IL, 2010.

19. Cruden, B. A., Brandis, A. M., and Prabhu, D. K., "Compositional Dependence of Radiance in CO2/N2/Ar Systems," AIAA 2013-2502.

20. Johnston, C. O., and Brandis, A. M., "Modeling of nonequilibrium CO Fourth-Positive and CN Violet emission in CO2-N2 gases," Journal of Quantitative Spectroscopy and Radiative Transfer, Vol. 149, 2014, pp. 303-317.

21. Depraz, S., Perrin, M. Y., and Soufiani, A., "Infrared emission spectroscopy of CO2 at high temperature. Part I: Experimental setup and source characterization," Journal of Quantitative Spectroscopy and Radiative Transfer, Vol. 113, No. 1, 2012, pp. 1-13. 
22. Depraz, S., Perrin, M. Y., Rivière, P., and Soufiani, A., "Infrared emission spectroscopy of CO2 at high temperature. Part II: Experimental results and comparisons with spectroscopic databases," Journal of Quantitative Spectroscopy and Radiative Transfer, Vol. 113, No. 1, 2012, pp. 14-25.

23. Milos, F., Chen, Y.-K., and Gokcen, T., "Non-Equilibrium Ablation of Phenolic Impregnated Carbon Ablator," 2010.

24. Mahzari, M., White, T. R., Braun, R., and Bose, D., "Inverse Estimation of the Mars Science Laboratory Entry Aerothermal Environment and Thermal Protection System Response," AIAA 2013-2780.

25. Mahzari, M., White, T., Braun, R., and Bose, D., "Preliminary Analysis of the Mars Science Laboratory's Entry Aerothermodynamic Environment and Thermal Protection System Performance," AIAA 2013-0185.

26. Milos, F. S., and Chen, Y.-K., "Ablation and Thermal Response Property Model Validation for Phenolic Impregnated Carbon Ablator," Journal of Spacecraft and Rockets, Vol. 47, No. 5, 2010, pp. 786-805.

27. Driver, D. M., Carballo, J. E., Beck, R., Prabhu, D., Santos, J. A., Cassell, A., Skokova, K., Tang, C., Hwang, H. H., Slimko, E., Willcockson, W., and Songer, J., "Arcjet Testing in Shear Environment for Mars Science Laboratory Thermal Protection System," Journal of Spacecraft and Rockets, Vol. 51, No. 4, 2014, pp. 1151-1166.

28. Driver, D., Olson, M., Barnhardt, M., MacLean, M., and MacLean, M., "Understanding High Recession Rates of Carbon Ablators Seen in Shear Tests in an Arc Jet," 2010.

29. Muppidi, S., Barnhardt, M., and Mansour, N. N., "Toward Ablative Material Response Coupling in DPLR," 2014. 